\title{
SIMULATIONS OF METHANE MOTION IN A VERTICAL COAL BED WHEN FILLED WITH WATER
}

\author{
Aleksey Bubenchikov ${ }^{1,2}$, Valentina Tsyrenova ${ }^{2}$, and Sevan Tsydypov ${ }^{2, *}$ \\ ${ }^{1}$ National Research Tomsk State University, 634050 Tomsk, Russia \\ ${ }^{2}$ Buryat State University, 670000 Ulan-Ude, Russia
}

\begin{abstract}
The present paper reviews pressure-gravitational movement of a gas and liquid medium in a vertical isotropic bed of a canonical rectangular shape with two permeability windows on the side borders. The work proposes a simple computing technology based on symmetric approximation of differential members and the Gauss-Seidel linearization which allows to significantly expand the range of the model's applicability from the standpoint of moisture saturation compared to traditional numerical.
\end{abstract}

\section{Introduction}

The output of methane from coal beds to the mine area and the surface layer of the atmosphere [1-4] and its combustion [5-7] present a certain danger to human life and activity. Therefore, the work directed on defining levels of methane concentration in case of flooding or releasing water from coal mines is not only appropriate, but also essential both in the environmental aspect and in terms of health and safety. The importance of this issue is proved by the recent tragic events in the coal mine "Severnaya".

In modern conditions, a correct prediction of methane distribution over the volume of a deposit should be based on an adequate and verified mathematical model of two-phase filtration in a coal bed and extensive geological information in the vicinity of the deposit. Along with this, the mathematical model must contain data on complex spatial architecture of mine workings.

The same as in mechanics of turbulent flows, the predictive function of a filtration model is higher if positive experience of its application is wider. Thus, along with the already implemented applications of the proposed mathematical model [1-4], in this paper, we estimate the effect of gravity on the dynamics of a two-phase medium in a vertical bed. Leaving aside the problem of fluid motion out of the porous space, i.e. in the area directly related to mine development, we will focus on pressure-gravitational movement of a gas and liquid medium in a vertical isotropic bed of a canonical rectangular shape with two permeability windows on the side borders. It is assumed that the wetting and the nonwetting phase each move in their channel systems. The movement of each of the phases

* Corresponding author: hudobina@mail2000.ru 
occurs under the influence of its own phase pressure and permeability of the bed with respect to each of the media is determined by its own permeability. In this case, the defining equations can be written in the form of the mass conservation laws for the gas and the liquid phases [8-10]:

$$
\begin{gathered}
\frac{\partial \rho^{G} m(1-s)}{\partial t}-\operatorname{div}\left(\frac{\mathrm{IK} k_{r}^{G}}{\mu^{G}} \rho^{G}\left(\vec{\nabla} p^{G}-\rho^{G} \vec{g}\right)\right)=q^{G}, \\
\frac{\partial \rho^{F} m s}{\partial t}-\operatorname{div}\left(\frac{\mathrm{IK} k_{r}^{F}}{\mu^{F}} \rho^{F}\left(\vec{\nabla} p^{F}-\rho^{F} \vec{g}\right)\right)=q^{F},
\end{gathered}
$$

here $\rho^{G}=\rho^{G}\left(p^{G}\right)=\rho^{G, 0} p^{G} / p^{0}$ is the density of the gaseous phase, $p_{0}$ is the atmospheric pressure; $m=m\left(p^{G}\right)$ is the porosity $\left(V_{n} / V, V_{\mathrm{n}}\right.$ are the pore volume); $s$ is the effective moisture saturation (in principle, varies from 0 to 1 ), IK is the permeability tensor, generally, fully-filled; $k_{r}^{G}=\sqrt{1-s}\left(1-s^{2}\right)$ is the relative permeability of the gas phase, $\mu_{G}$ is the gas viscosity, $q_{G}$ is the source member in the balance of the gas phase, $q_{F}$ is the source member in the fluid balance; $\rho^{F}=\rho^{F, 0} p^{F} / p^{0}, \rho^{F, 0}$ is the density of the fluid at atmospheric pressure; $k_{r}^{F}=\sqrt{s}\left(1-\sqrt{1-s^{2}}\right)^{2}$ is the relative permeability for the liquid phase, $\mu_{F}$ is the viscosity of the fluid; $p^{F}=p^{G}-p^{c}(s) ; p^{c}(s)=p^{d} \sqrt{1-s^{2}} / s$ is the capillary pressure, $p^{d}$ is the threshold pressure, while $p^{d}=\rho^{F} g / \alpha$, where $\alpha$ is the parameter characterizing the porous medium.

\section{Calculation results}

The equations of mass balance of the liquid and the gaseous phases which include densities or pressures of the phases are non-linear equations. Furthermore, the coefficients at the derivatives in these equations at particular points of the area may become zero. This peculiarity creates obstacles to the use of specific numerical techniques. Typically, these obstacles are overcome at the cost of narrowing the range of moisture saturation changes. However, in this paper we propose a numerical algorithm which allows carrying out calculations in the whole range of moisture saturation changes.

The numerical algorithm is based on the use of the simplest method of calculating nonlinear coefficients by deflecting the included designed parameters to the previous iteration layer without using gradient methods for accelerating the convergence. The efficiency of the constructed algorithm is confirmed by good agreement of the calculation results with the analytical distribution of Buckley-Leverett for plane-radial filtration flow. 


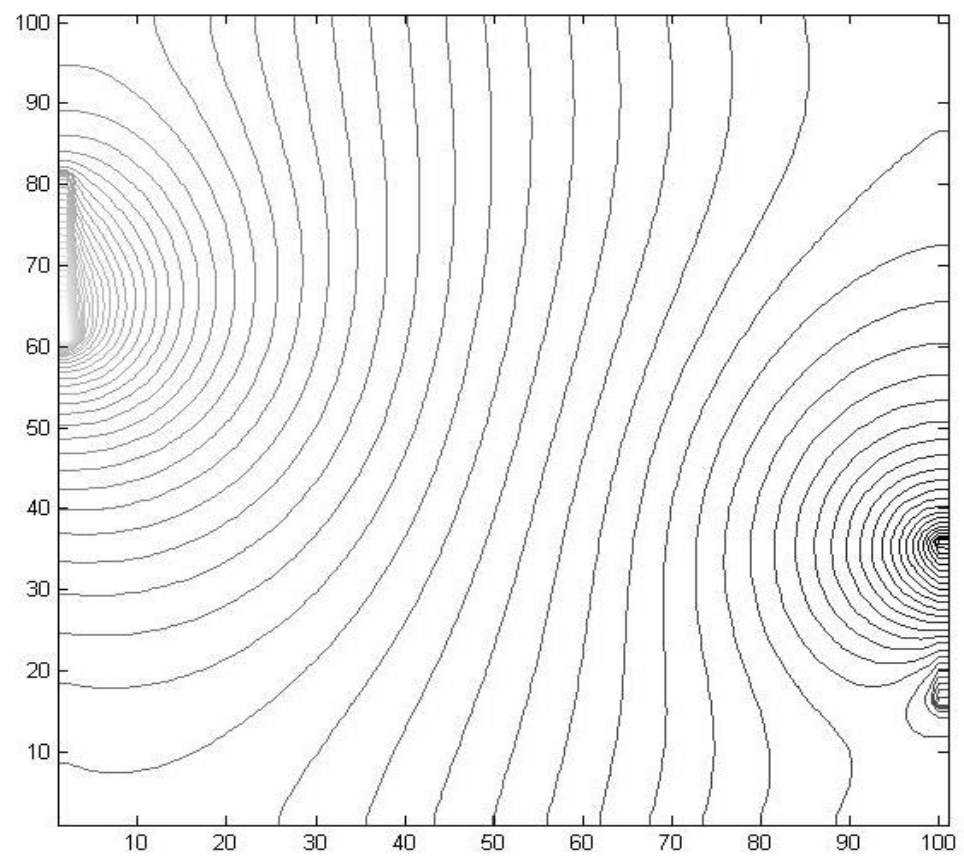

Fig. 1. Lines of equal pressure in gas phase.

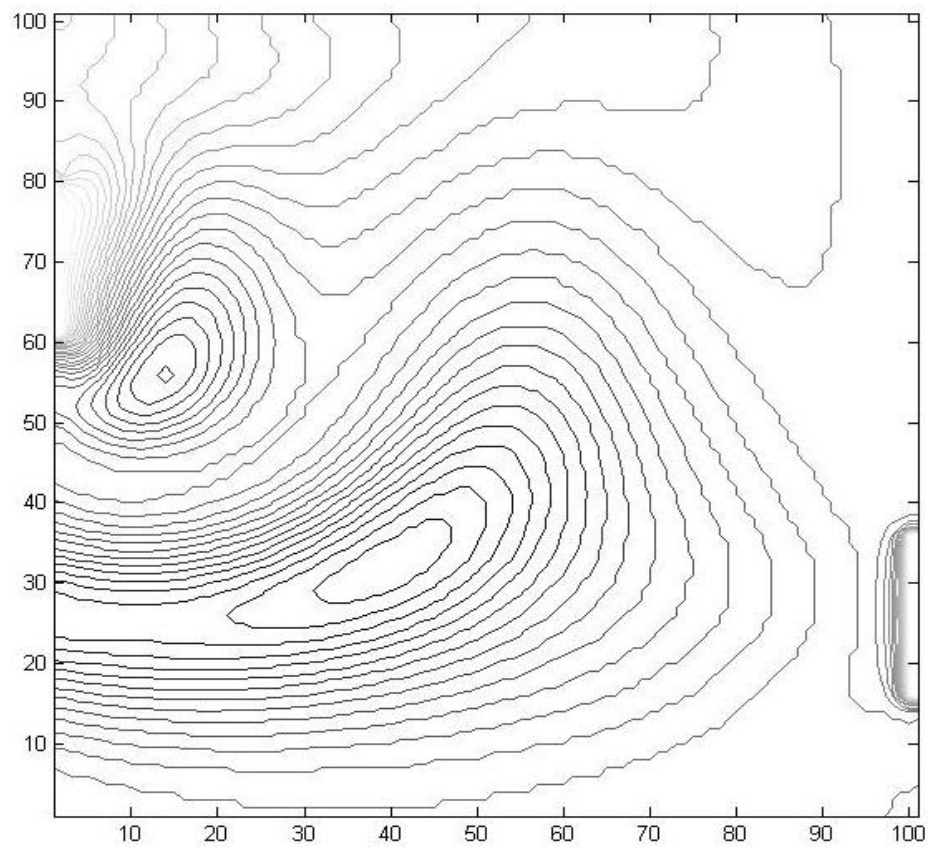

Fig. 2. Isolines of fluid pressure distribution.

The results shown in fig.1, 2 correspond to the case of steady flow which is obtained from the initial state of rest by the establishment method. At a certain point in time, taken as the start, the hydraulic fluid pressure increases in the permeability window on the left. Thereafter, movement of both phases begins. Calculations show that the gas phase appears 
to be more mobile at the stage of establishment, but, ultimately, the gas distribution pattern in the porous space becomes easier.

\section{Acknowledgments}

This paper was written within the frame of Competitiveness Improvement Program of Tomsk State University.

\section{References}

1. D.O.Dil, A.M.Bubenchikov, Vestnik TGU. Meh. Matem. 6, 26 (2013) [in Russian]

2. D.O.Dil, A.M.Bubenchikov, Vestnik TGU. Meh. Matem. 5, 25 (2013) [in Russian]

3. D. O. Dil, A. M. Bubenchikov, Key Eng. Mat. 685 (2016)

4. A.M. Bubenchikov, V.B. Tsyrenova, S.G. Tsydypov, Vestnik TGU. Meh. Matem. 1 (2014) [in Russian]

5. O.V. Matvienko, A.M. Bubenchikov, J. of Eng. Phys. and Therm. (to be published)

6. A.M. Bubenchikov, V.A. Korobitsyn, D.V. Korobitsyn, P.P. Kotov, Y.I. Shokin, J. Comp. Math. and Math. Phys. 54, 7 (2014)

7. O.V. Matvienko, A.I. Baigulova, A.M. Bubenchikov, J. of Eng. Phys. and Therm. 87, $6(2014)$

8. C. Shikuo, Y. Tianhong, W. Chenhui, Mater. of Int. Symp., China (2010)

9. M.Th. Van Genuchten, Soil Sci. Soc. Am. J. 44 (1980)

10. M.G. Schaap, M.Th. Van Genuchten, Vadose Zone J. 5 (2006) 